\title{
Refractory neutrophils and monocytes in patients with inflammatory bowel disease after repeated bouts of prolonged exercise
}

\author{
Roy Spijkerman ${ }^{1}$, Lilian Hesselink ${ }^{1}$, Carlo Bertinetto ${ }^{2}$, Coen Bongers ${ }^{3}$, Falco Hietbrink ${ }^{1}$, \\ Nienke Vrisekoop ${ }^{1}$, Luke Leenen ${ }^{1}$, Maria Hopman ${ }^{3}$, Jeroen Jansen ${ }^{2}$, and Leo Koenderman ${ }^{1}$ \\ ${ }^{1} \mathrm{UMC}$ Utrecht \\ ${ }^{2}$ Radboud University Nijmegen \\ ${ }^{3} \mathrm{UMC}$ Sint Radboud
}

August 28, 2020

\begin{abstract}
Rationale Neutrophils and monocytes are key immune effector cells in inflammatory bowel disease (IBD) that is associated with chronic inflammation in the gut. Patients with stable IBD who exercise have fewer flare-ups, but no underlying mechanism has been identified. Therefore, the aim of this study was to compare the responsiveness of these innate immune cells after repeated bouts of prolonged exercise in IBD patients and controls. Methods Patients with IBD and age- and gender-matched healthy controls were recruited from a cohort of walkers participating in a 4-day walking event. Blood analysis was performed at baseline and after 3 days of walking. Responsiveness to the bacterial/mitochondrial N-Formylmethionine-leucyl-phenylalanine (fMLF) was tested in granulocytes and monocytes by measuring the expression of activation markers after adding this stimulus to whole blood Results In total 38 participants ( $54 \pm 12$ years) were included in this study: 19 walkers with and 19 walkers without IBD. After 3 days of prolonged exercise, a significant increase in responsiveness to fMLF was observed in all participants irrespective of disease. However, IBD patients showed significantly smaller increase in neutrophils $(p=0.010 ; p=0.030 ; p=0.010$, respectively) and monocytes $(\mathrm{p}=0.001 ; \mathrm{p}=0.008 ; \mathrm{p}=0.005$, respectively), compared to controls. Conclusions Increased responsiveness of neutrophils and monocyte to fMLF was demonstrated after repetitive bouts of prolonged exercise. Interestingly, this exercise was associated with relative refractoriness of both neutrophils and monocytes in IBD patients. These refractory cells might create a lower inflammatory state in the intestine providing a putative mechanism for the decrease in flare-ups in IBD patients after repeated exercise.
\end{abstract}

Refractory neutrophils and monocytes in patients with inflammatory bowel disease after repeated bouts of prolonged exercise

Roy Spijkerman ${ }^{1,2,3}$, Lillian Hesselink ${ }^{1,2}$, Carlo Bertinetto ${ }^{4}$, Coen CWG Bongers ${ }^{5}$, Falco Hietbrink ${ }^{1}$, Nienke Vrisekoop ${ }^{2,3}$, Luke PH Leenen ${ }^{1}$, Maria TE Hopman ${ }^{5}$, Jeroen J Jansen ${ }^{4}$, Leo Koenderman ${ }^{2,3}$.

${ }^{1}$ Department of Trauma Surgery, University Medical Center Utrecht (Heidelberglaan 100, 3584 CX), Utrecht, The Netherlands.

${ }^{2}$ Center for Translational Immunology (CTI), University Medical Center Utrecht (Heidelberglaan 100, 3584 CX), Utrecht, The Netherlands. 
${ }^{3}$ Department of Respiratory Medicine, University Medical Center Utrecht (Heidelberglaan 100, 3584 CX), Utrecht, The Netherlands

${ }^{4}$ Institute for Molecules and Materials (Analytical Chemistry), Radboud University (Heyendaalseweg 135, 6525 AJ), Nijmegen, The Netherlands

${ }^{5}$ Department of Physiology Radboud Institute for Health Sciences (RIHS), Radboud university medical center (Geert Grooteplein Zuid 10, 6525 GA), Nijmegen, The Netherlands.

Correspondence to:Leo Koenderman, Department of Respiratory Medicine and Center for Translational Immunology, University Medical Center Utrecht (Heidelberglaan 100, 3584 CX), Utrecht, The Netherlands E-mail: l.koenderman@umcutrecht.nl

Funding: The AQUIOS CL ${ }^{\circledR}$ "load \& go" flow cytometer is provided by the company Beckman Coulter Life Sciences, Miami, FL, USA. All authors declare that there are no other competing interests. This article was supported by a grant of the Netherlands Organization for Scientific Research (NWO) in the framework of the "Startimpulse Measurement \& Detection of healthy behavior" Dutch National Research Agenda.

Conflicts of interest: All authors declare that they do not have potential conflicts of interest.

Study highlights:

1. Patients with stable IBD who perform exercise have significantly fewer flare-ups of disease

2. Relative refractory neutrophils and monocytes were demonstrated in peripheral blood of IBD patients after prolonged exercise.

3. Increased knowledge on the pathophysiological mechanism on the effect of exercise on IBD facilitates future therapy to prevent flare-ups.

AbstractRationale Neutrophils and monocytes are key immune effector cells in inflammatory bowel disease (IBD) that is associated with chronic inflammation in the gut. Patients with stable IBD who perform exercise have significantly fewer flare-ups of the disease, but no underlying mechanism has been identified. Therefore, the aim of this study was to compare the responsiveness of these innate immune cells after repeated bouts of prolonged exercise in IBD patients and controls.

Methods Patients with IBD and age- and gender-matched healthy controls were recruited from a cohort of walkers participating in a 4-day walking event. Blood analysis was performed at baseline and after 3 days of walking. Responsiveness to the bacterial/mitochondrial N-Formylmethionine-leucyl-phenylalanine (fMLF) was tested in granulocytes and monocytes by measuring the expression of activation markers after adding this stimulus to whole blood

Results In total 38 participants ( $54 \pm 12$ years) were included in this study: 19 walkers with and 19 walkers without IBD. After 3 days of prolonged exercise, a significant increase in responsiveness to fMLF was observed in all participants irrespective of disease. However, IBD patients showed significantly smaller increase in neutrophils $(\mathrm{p}=0.010 ; \mathrm{p}=0.030 ; \mathrm{p}=0.010$, respectively) and monocytes $(\mathrm{p}=0.001 ; \mathrm{p}=0.008 ; \mathrm{p}=0.005$, respectively), compared to controls.

\section{Conclusions}


Increased responsiveness of neutrophils and monocyte to fMLF was demonstrated after repetitive bouts of prolonged exercise. Interestingly, this exercise was associated with relative refractoriness of both neutrophils and monocytes in IBD patients. These refractory cells might create a lower inflammatory state in the intestine providing a putative mechanism for the decrease in flare-ups in IBD patients after repeated exercise

\section{Keywords:}

Exercise, neutrophil, monocyte, inflammatory bowel disease, flare-ups.

\section{Introduction}

Inflammatory bowel disease (IBD) is a chronic active inflammatory condition of the digestive tract. It includes both Crohn's disease, which can affect all parts of the digestive system, and ulcerative colitis, which mainly affects the colon and rectum(1). IBD is caused by an imbalance in the three major components that contribute to gut homeostasis: the tissue, intestinal microbes, and immune cells $(2,3)$. During intestinal inflammation, intestinal cells, monocyte-derived macrophages and $\mathrm{T}$ helper 17 cells stimulate the recruitment of polymorphonuclear leukocytes $(\mathrm{PMN})$ and monocytes through production of chemokines and cytokines $(2,4,5)$. The resulting gradients of these mediators stimulate massive amounts of blood PMNs and monocytes to transverse the endothelium and reach the lamina propria of the intestinal tract, where PMNs and monocytes contribute to the inflammation $(2,6,7)$.

Exercise has a beneficial effect on IBD, likely by changing the three major components that contribute to gut homeostasis (epithelium, intestinal microbes, and immune cells) (8-10). A large observational study showed that patients with stable IBD were significantly less likely to develop active disease in six months when undertaking activities(11). However, the underlying pathophysiologic mechanisms have not been elucidated.

Several studies have shown an altered cellular adaptive immune response (T-cell changes, Natural killer cell activity, salivary IgA production), cytokine production (IL6, IL8, IL10) and innate immune responses (granulocyte cell count, granulocyte respiratory burst, neutrophil/lymphocyte ratio and macrophage activity) for several hours to days, during recovery from prolonged exercise(12-15). However, repeated bouts of prolonged moderate exercise result in acute inflammation the first day, followed by a normalization/adaptation of the immune response every consecutive day(15).

PMNs and monocytes play a critical role in the pathophysiology of $\operatorname{IBD}(2,16,17)$. Responsiveness of PMNs and monocytes can be tested byin vitro, adding the bacterial/mitochondrial stimulus N-Formylmethionineleucyl-phenylalanine (fMLF)(18-20). Testing the responsiveness of innate immune cells to fMLF is valuable to the understanding of functional cellular activity in several diseases(21-23). It is known that cellular responsiveness to fMLF is significantly increased in patients with IBD flare-ups(24). Testing PMN and monocyte responsiveness to fMLF might help in understanding the role of PMNs and monocytes in exercise immunology. Therefore, the aim of this study was to compare the responsiveness of these innate immune cells after repeated bouts of prolonged exercise in IBD patients and controls.

\section{Methods}

\section{Study population}

A total of 19 patients with IBD were included in the study. Inclusion criteria for all IBD patients were age [?]18 years and diagnosis of Crohn's disease or ulcerative colitis made by a gastroenterologist. IBD patients were not included when they used specific biologicals (infliximab, adalimumab, golimumab, ustekinumab) as these might change the immune response. All patients completed a distance of 30,40 or $50 \mathrm{~km}$ per day on three consecutive days at a self-selected pace. As a control group, a total of 19 age- and gender-matched healthy controls were recruited who also underwent the same repeated exercise bouts. This study was approved by the Medical Ethical Committee region Arnhem-Nijmegen (CMO registration number: 20195375) and was registered at trialregister.nl as NL7872. All participants provided written informed consent prior to participation. This study was conducted in accordance with the Declaration of Helsinki.

\section{Baseline measurements}


Baseline data were collected 1 or 2 days before the start of the event, after a minimum resting period of $24 \mathrm{~h}$. At baseline, body height and weight (Seca 888 scale, Hamburg, Germany) were measured to calculate body mass index (BMI). All participants completed a general questionnaire on demographics, level of education, smoking and medication use, and the validated Short Questionnaire to Assess Health enhancing physical activity (SQUASH)(25). IBD walkers completed an extended general questionnaire with additional questions on type and extent of IBD, age of disease onset, number of flare-ups and previous IBD-related surgeries. Participants' heart rate was measured every $5 \mathrm{~km}$ during the first exercise day using a 2-channel ECG chest band 130 system. Heart rate (HR) was used to estimate exercise intensity as a percentage of the maximum $\mathrm{HR}$ (exercise intensity = measured HR / expected maximal HR x 100\%), where expected max $\mathrm{HR}=208$ $-132(0.7 \times$ age $))(26)$.

\section{Blood sampling}

Venous blood was drawn at baseline and after three days of repeated bouts of exercise. A 4 mL Vacutainer ${ }^{(\mathrm{r})}$ sodium heparin blood tube (Becton Dickinson, Oakville, ON, USA) was drawn, which was directly inserted in the automated AQUIOS CL ${ }^{(r)}$ "Load \& Go" flow cytometer (Beckman Coulter, Miami, FL, USA).

\section{Flow cytometry analysis}

The AQUIOS CL combines robotic automated sample preparation with an analysis of cells using flow cytometry (27). The AQUIOS CL has one $488 \mathrm{~nm}$ diode laser, two light scatter channels (forward scatter and side scatter), 5 fluorescence channels, and an electronic volume measure. Absolute leukocyte count was based on an electronic-volume measurement. A cassette filled with blood tubes was placed in the machine and the barcodes of the samples were saved. After automatic blood mixing, the samples were cap-pierced, and 43 $\mu \mathrm{L}$ was pipetted into a 96-deep wells plate, that is used for antibody staining. The wells plate contained the bacterial/mitochondrial-derived stimulus formyl-methionyl-leucyl-phenylalanine (fMLF) (Sigma Aldrich, St. Louis, MO, USA) with an end concentration of $10^{-6} \mathrm{M}$. Details of antibody mixes are provided in the online supplement. Consecutively, $18 \mu \mathrm{L}$ of a monoclonal antibody mix bound to different fluorescent labels was added to the 96 -wells plate. After $15 \mathrm{~min}$ of incubation, the blood was lysed using $335 \mu \mathrm{L}$ of lysing reagents $\mathrm{A}$ and $100 \mu \mathrm{L}$ of lysing reagent B. Lysing reagent $\mathrm{A}$ is a cyanide-free lytic reagent that lyses red blood cells. Lysing reagent B slows the reaction caused by reagent A and preserved the white blood cells for measurement in the flow cell. Finally, $100 \mu \mathrm{L}$ of the prepared sample was aspirated for analysis.

A customized antibody mix was made specifically for this research purpose. This panel consisted of CD35FITC (clone J3.D3, Beckman Coulter, Miami, FL, USA), CD16-PE (clone 3G8, Beckman Coulter), CD62LECD (clone DREG56, Beckman Coulter), CD11b-PC5 (clone Bear1, Beckman Coulter) and CD10-PC7 (clone ALB1, Beckman Coulter).

The .lmd data files were exported from the AQUIOS CL ${ }^{\circledR}$ and imported into FlowJo ${ }^{\circledR}$ analysis software (Tree Star Inc., Ashland, OG, USA). The gating strategy is shown in supplemental figure 1. Granulocytes and monocytes were gated based on forward scatter and side scatter. Neutrophils and eosinophils in the granulocyte gate were identified based on positivity or negativity of CD16 expression. The median fluorescent intensity (MFI) of CD35, CD11b, CD62L and CD10 on gated neutrophils was used to describe the measure neutrophil and monocyte responsiveness to fMLF.

\section{Statistical analysis}

The statistical analyses were conducted in SPSS 26 (SPSS Inc., Chicago, IL, USA). Graphs were created with GraphPad Prism version 7 (GraphPad software inc., San Diego, CA, USA). Data were checked for normality with use of the D'Agostina \& Pearson test and visual inspection of Q-Q plots. Statistical significance was defined as a p-value $<0.05$. Normally distributed data were presented as mean with standard deviation (SD) and significance was tested by a t-test. Non-normally distributed data were presented as median with interquartile range (IQR) and significance was tested by a Mann-Whitney U test. Dichotomous data were shown as an absolute count with the percentage of the total, in which differences were tested with the Pearson Chi-square test. 


\section{Results}

In total 38 participants were included in this study, of whom 19 IBD walkers, 19 non-IBD walkers. One IBD walker fell during exercise on day 1 and dropped out, he was excluded from all statistical analysis. Clinical disease characteristics of IBD patients are shown in Table 1. The IBD study groups consisted of 8 patients with Crohn's disease and 10 patients with ulcerative colitis. The mean age of diagnosis was $33 \pm$ 13 years. One year before the walking event, a total of 8 (44\%) IBD patients had no flare-ups, 6 patients had one to two flare-ups and 4 patients had three of more flare-ups. Baseline characteristics of both between the IBD walkers and the age/gender-matched control group are shown in table 2 . The mean age of the study population was $54 \pm 12$ years and the average BMI of all participants was $26.0 \pm 3.8 \mathrm{~kg} / \mathrm{m} 2$. In each group, 11 participants were female. No significant differences were found between IBD walkers and non-IBD walkers regarding participant characteristics, except for training distance during the last two weeks before the walking event (IBD walkers vs non-IBD walkers, $\mathrm{p}=0.03$ ).

Responsiveness to fMLF, determined by flow cytometric determination of innate immune markers, is shown in Figure 1. The cellular responsiveness to fMLF is shown as the Median Fluorescent Intensity(MFI) of innate immune markers: Neprilysine (CD10) (Figure 1A), Complement receptor 3 (CD11b) (Figure 1C-D), Complement receptor 1 (CD35) (Figure $1 \mathrm{E}-\mathrm{G}$ ) and L-selectin (CD62L) (Figure 1H-J).

Smaller increased responsiveness to fMLF was shown after 3 days of prolonged exercise in the expression of the activation markers CD11b and CD35 in IBD walkers compared to non-IBD walkers in neutrophils $(\mathrm{p}=0.01 ; \mathrm{p}=0.03)$ and monocytes $(\mathrm{p}=0.001 ; \mathrm{p}=0.008)$. No difference in CD11b and CD35 expression was demonstrated between the study groups at baseline measurements. No difference in responsiveness was seen in the cell adhesion receptor CD62L in neutrophils, monocytes and eosinophils between the study groups. The maturation/activation marker CD10 showed similar smaller increased responsiveness to fMLF after 3 days of repeated prolonged exercise in IBD walkers compared to non-IBD walkers in neutrophils $(\mathrm{p}=0.005)$. However, already at day 0 , a lower expression of CD10 was demonstrated in the neutrophil compartment of IBD walkers compared to non-IBD walkers $(\mathrm{p}=0.01)$.

\section{Discussion}

This study investigated the effect of 3 repeated bouts of prolonged exercise on neutrophils, eosinophils and monocytes in patients with inflammatory bowel disease. We used automated point-of-care flow cytometry in a field laboratory with an integrated system to test the fMLF responsiveness of eosinophils, neutrophils and monocytes. We found that IBD patients demonstrated a smaller increase in neutrophil and monocyte responsiveness in activation markers CD35 and CD11b towards an inflammatory stimulus after repeated walking, compared to non-IBD walkers. A decreased neutrophil responsiveness was also observed in the activation/maturation marker CD10.

In previous studies on repeated prolonged exercise, conducted during the same walking event, a general increase in cytokines and direct innate immune activation was found after the first day in all tested participants(15). After the second and third day a normalization/adaptation of the innate immune system was demonstrated. The present study showed no difference between controls and IBD patients in responsiveness of neutrophils, eosinophils and monocytes to fMLF during the first 2 days of exercise (results not shown). Only at day 3 increased responsiveness of neutrophil and monocytes was found in all walkers (IBD and nonIBD), but was significantly less increased in IBD patients pointing. The underlying mechanism remains to be established, but it is tempting to speculate that increased responsiveness is the result of a priming effect in response to damage-associated molecular patterns (DAMPs) originating from mild-exercise-induced tissue damage during the first 2 days. Apparently, this situation leads to a suppression of the proinflammatory response in IBD patients.

The neutrophil activation markers CD35, CD11b and CD10 showed the same significant differences between the study groups after 3 days of repeated bouts of prolonged exercise. The complement receptors CD11b and CD35 showed no difference in expression between participants with IBD and without IBD at day 0. However, CD10 was also significantly different at day 0 , which might be an indirect effect of the additional 
role of maturation marker (28). Patients with IBD are characterized by chronic inflammation, which might associate to an overall younger neutrophil compartment (left shift) in the bloodstream(2).

In diseases with hypo inflammation of the innate immune system, a decreased neutrophil responsiveness in the peripheral blood is associated with worse clinical outcomes (infectious complications and sepsis) $(22,23,29)$. In diseases associated with hyper inflammation, decreased neutrophil responsiveness is associated with better clinical outcomes (allergic/auto-immune diseases)(30). Three days of exercise in IBD patients is associated with the occurrence of less increased responsive neutrophils and monocytes in the peripheral blood. Possibly explaining the better clinical outcomes in IBD patients.

In conclusion, increased responsiveness of neutrophils and monocyte to fMLF was demonstrated after repetitive bouts of prolonged exercise. Interestingly, this exercise was associated with relative refractoriness of both neutrophils and monocytes in IBD patients. These refractory cells might create a lower inflammatory state in the intestine, providing a putative mechanism for the decrease in flare-ups in IBD patients after repeated exercise.

\section{Author contribution:}

All authors made substantial contributions to the conception of the work. RS, LH and CCGWB worked on data acquisition. RS, LH and CB performed the data analysis. All authors worked on the interpretation of data. RS made a substantial contribution to the drafting of the work. All authors made a substantial contribution to revising the work critically for relevant intellectual content. All authors approved the final version to be published. All authors agree to be accountable for all aspects of the work in ensuring that questions related to the accuracy or integrity of any part of the work are appropriately investigated and resolved.

\section{Acknowledgements:}

For technical support, we want to thank Paul van Hoof, Roelof-Jan van der Lei, Geert Weijers, Andreas Boehmler and Markus Kaymer from the Beckman Coulter team. We also want to thank the whole team of the Nijmegen Exercise Study for their help with blood drawing and collection of the questionnaire data. We want to thank Tjarda Tromp, for coordinating the blood drawing procedures.

\section{References}

1. Baumgart DC, Sandborn WJ. Inflammatory bowel disease: clinical aspects and established and evolving therapies. Lancet. 2007;369(9573):1641-57.

2. Fournier BM, Parkos CA. The role of neutrophils during intestinal inflammation. Mucosal Immunol. $2012 ; 5: 354-366$.

3. Maloy KJ, Powrie F. Intestinal homeostasis and its breakdown in inflammatory bowel disease. Nature. 2011;474:298-306.

4. Ajuebor MN, Das AM, Virág L, Flower RJ, Szabó C, Perretti M. Role of resident peritoneal macrophages and mast cells in chemokine production and neutrophil migration in acute inflammation: evidence for an inhibitory loop involving endogenous IL-10. J Immunol. 1999;162(3):1685-91.

5. Witowski J, Pawlaczyk K, Breborowicz A, Scheuren A, Kuzlan-Pawlaczyk M, Wisniewska J, et al. IL-17 Stimulates Intraperitoneal Neutrophil Infiltration Through the Release of GRO $\alpha$ Chemokine from Mesothelial Cells. J Immunol. 2000;165(10):5814-21.

6. Kucharzik T, Hudson JT, Lügering A, Abbas JA, Bettini M, Lake JG, et al. Acute induction of human IL-8 production by intestinal epithelium triggers neutrophil infiltration without mucosal injury. Gut. 2005;54(11):1565-72.

7. MacDermott RP. Chemokines in the inflammatory bowel diseases. J Clin Immunol. 1999;77:44-9. 
8. Codella R, Luzi L, Terruzzi I. Exercise has the guts: How physical activity may positively modulate gut microbiota in chronic and immune-based diseases. Dig Liver Dis. 2018;50(4):331-41.

9. Bilski J, Mazur-Bialy A, Brzozowski B, Magierowski M, Zahradnik-Bilska J, Wójcik D, et al. Can exercise affect the course of inflammatory bowel disease? Experimental and clinical evidence. Pharmacol Reports. 2016;68(4):827-36.

10. Monda V, Villano I, Messina A, Valenzano A, Esposito T, Moscatelli F, et al. Exercise modifies the gut microbiota with positive health effects. Oxid Med Cell Longev. 2017;3831972.

11. Jones PD, Kappelman MD, Martin CF, Chen W, Sandler RS, Long MD. Exercise decreases risk of future active disease in patients with inflammatory bowel disease in remission. Inflamm Bowel Dis. 2015;21(5):106371.

12. Peake JM, Gatta P Della, Suzuki K, Nieman DC. Cytokine expression and secretion by skeletal muscle cells: Regulatory mechanisms and exercise effects. Exerc Immunol Rev. 2015;21:8-25.

13. Simpson RJ, Kunz H, Agha N, Graff R. Exercise and the Regulation of Immune Functions. Prog Mol Biol Transl Sci. 2015;135:355-80.

14. Brown M, McClean CM, Davison GW, Brown JCW, Murphy MH. The acute effects of walking exercise intensity on systemic cytokines and oxidative stress. Eur J Appl Physiol. 2018;18(10):2111-20.

15. Terink R, Bongers CCWG, Witkamp RF, Mensink M, Eijsvogels TM, Klein Gunnewiek JMT, et al. Changes in cytokine levels after prolonged and repeated moderate intensity exercise in middle-aged men and women. Transl Sport Med. 2018;1 (3):110-9.

16. Zhou GX, Liu ZJ. Potential roles of neutrophils in regulating intestinal mucosal inflammation of inflammatory bowel disease. J Dig Dis. 2017;18(9):495-503.

17. Kühl AA, Kakirman H, Janotta M, Dreher S, Cremer P, Pawlowski NN, et al. Aggravation of Different Types of Experimental Colitis by Depletion or Adhesion Blockade of Neutrophils. Gastroenterology. 2007;133(6):1882-92.

18. Marasco WA, Phan SH, Krutzsch H, Showell HJ, Feltner DE, Nairn R, et al. Purification and identification of formyl-methionyl-leucyl-phenylalanine as the major peptide neutrophil chemotactic factor produced by Escherichia coli. J Biol Chem. 1984;259(9):5430-9.

19. Hesselink L, Spijkerman R, Van Wessem KJP, Koenderman L, Leenen LPH, Huber-Lang M, et al. Neutrophil heterogeneity and its role in infectious complications after severe trauma. World J Emerg Surg. 2019;29:14:24.

20. Spijkerman R, Hesselink L, Bongers S, van Wessem KJP, Vrisekoop N, Hietbrink F, et al. Point-of-Care Analysis of Neutrophil Phenotypes: A First Step Toward Immuno-Based Precision Medicine in the Trauma ICU. Crit Care Explor. 2020;2(7):e0158.

21. Pillay J, Ramakers BP, Kamp VM, Loi ALT, Lam SW, Hietbrink F, et al. Functional heterogeneity and differential priming of circulating neutrophils in human experimental endotoxemia. J Leukoc Biol. 2010 $\mathrm{Jul} ; 88(1): 211-20$.

22. Hietbrink F, Koenderman L, Althuizen M, Pillay J, Kamp V, Leenen LPH. Kinetics of the Innate Immune Response After Trauma. Shock. 2013;40(1):21-7.

23. Groeneveld KM, Koenderman L, Warren BL, Jol S, Leenen LPH, Hietbrink F. Early decreased neutrophil responsiveness is related to late onset sepsis in multitrauma patients: An international cohort study. PLoS One. 2017;12 (6):e0180145.

24. Anton PA, Targan SR, Shanahan F. Increased neutrophil receptors for and response to the proinflammatory bacterial peptide formyl-methionyl-leucyl-phenylalanine in Crohn's disease. Gastroenterology. 1989;97 
(1):20-8.

25. Wendel-Vos GCW, Schuit AJ, Saris WHM, Kromhout D. Reproducibility and relative validity of the short questionnaire to assess health-enhancing physical activity. J Clin Epidemiol. 2003;56(12):1163-9.

26. Tanaka H, Monahan KD, Seals DR. Age-predicted maximal heart rate revisited. J Am Coll Cardiol. 2001;37 (1):153-6.

27. Spijkerman R, Hesselink L, Hellebrekers P, Vrisekoop N, Hietbrink F, Leenen LPH, et al. Automated flow cytometry enables high performance point-of-care analysis of leukocyte phenotypes. J Immunol Methods. 2019 Aug;474:112646.

28. Marini O, Costa S, Bevilacqua D, Calzetti F, Tamassia N, Spina C, et al. Mature CD10+ and immature CD10- neutrophils present in G-CSF-treated donors display opposite effects on T cells. Blood. 2017;129(10):1343-56.

29. Edelman JJB, Fung YL, Pennings GJ, Reddel CJ, Bannon PG, Bayfield MS, et al. Off-pump coronary artery bypass surgery induces prolonged alterations to host neutrophil physiology. Shock. 2013;39(2):149-54.

30. Kanters D, ten Hove W, Luijk B, van Aalst C, Schweizer RC, Lammers JWJ, et al. Expression of activated FcrRII discriminates between multiple granulocyte-priming phenotypes in peripheral blood of allergic asthmatic subjects. J Allergy Clin Immunol. 2007;120 (5):1073-81.

\section{Figure legends}

Figure 1: Responsiveness of innate immune cells to the bacterial/mitochondrial stimulus fMLF in IBD $(N=18)$ versus non-IBD patients $(n=19)$. Responsiveness is determined by flowcytometric analysis of the cellular activation markers CD10 (A), CD11b(B-D), CD35 (E-G) and the adhesion marker CD62L(H-J) . MFI is compared between study groups at day 0 and after 3 consecutive days of prolonged moderate exercise with the Mann-Whitney U test. Data are presented as scatter plot with median and interquartile range. Abbreviations: $I B D=$ inflammatory bowel disease; $M F I=$ Median fluorescent intensity; $A U=$ arbitrary units.

\begin{tabular}{ll}
\hline & IBD (n=18) \\
\hline Age of diagnosis (years) & $33 \pm 13$ \\
Crohn's disease / Ulcerative colitis & $8(44 \%) / 10(66 \%)$ \\
$\begin{array}{l}\text { Disease location Crohn's disease (n (\%)) } \\
\text { Small bowel }\end{array}$ & $2(25 \%)$ \\
Colon & $2(25 \%)$ \\
Small bowel \& colon & $2(25 \%)$ \\
Unknown & $2(25 \%)$ \\
Disease location ulcerative colitis (n (\%)) & \\
Pancolon & $2(20 \%)$ \\
Hemicolon & $3(30 \%)$ \\
Rectum & $5(50 \%)$ \\
Flare-ups last 12 months (n (\%)) & \\
None & $8(44 \%)$ \\
1-2 flare-ups & $6(33 \%)$ \\
3-4 flare-ups & $2(11 \%)$ \\
More than 4 flare-ups & $2(11 \%)$ \\
Treatment of IBD (n (\%)) & \\
Receiving IBD medication & $11(61 \%)$ \\
5- $A S A$ oral & $9(50 \%)$ \\
5- $A S A$ rectal & $4(22 \%)$ \\
Corticosteroids oral & $4(22 \%)$ \\
&
\end{tabular}




\begin{tabular}{ll}
\hline & IBD $(\mathbf{n}=\mathbf{1 8})$ \\
\hline Corticosteroids rectal & $2(11 \%)$ \\
Other Immunosuppressants & $1(6 \%)$ \\
No use of IBD medication & $7(39 \%)$ \\
Received surgery for IBD & $1(6 \%)$ \\
\hline
\end{tabular}

Table 1: Disease characteristics of patients with inflammatory bowel disease . Continuous data are shown as median (IQR) and dichotomous data are shown as an absolute amount (percentage).

Abbreviations: $I B D=$ inflammatory bowel disease; $A S A=5$-aminosalicylic acid.

\begin{tabular}{|c|c|c|}
\hline & IBD $(n=18)$ & Non-IBD $(n=19)$ \\
\hline Age (years) & $54 \pm 11$ & $54 \pm 14$ \\
\hline Male n $(\%) /$ female $\mathrm{n}(\%)$ & $8(39 \%) / 11(61 \%)$ & $8(42 \%) / 11(58 \%)$ \\
\hline Body mass index $\left(\mathrm{kg} / \mathrm{m}^{2}\right)$ & $25.7 \pm 3.8$ & $26.0 \pm 4.5$ \\
\hline \multicolumn{3}{|l|}{ Smoking (n (\%)) } \\
\hline Never & $9(50 \%)$ & $13(68 \%)$ \\
\hline Current & $1(6 \%)$ & $0(0 \%)$ \\
\hline Former & $8(44 \%)$ & $6(32 \%)$ \\
\hline \multicolumn{3}{|l|}{$\begin{array}{l}\text { Walking distance per day } \\
(\mathrm{n}(\%))\end{array}$} \\
\hline $30 \mathrm{~km}$ & $5(28 \%)$ & $2(11 \%)$ \\
\hline $40 \mathrm{~km}$ & $11(61 \%)$ & $13(68 \%)$ \\
\hline $50 \mathrm{~km}$ & $2(11 \%)$ & $4(21 \%)$ \\
\hline \multicolumn{3}{|l|}{$\begin{array}{l}\text { Average walking speed per } \\
\text { day }(\mathrm{km} / \mathrm{h})\end{array}$} \\
\hline Day $1(\mathrm{~km} / \mathrm{h})$ & $4.5 \pm 0.8$ & $4.4 \pm 0.7$ \\
\hline Day $2(\mathrm{~km} / \mathrm{h})$ & $4.4 \pm 1.0$ & $4.4 \pm 0.8$ \\
\hline Day $3(\mathrm{~km} / \mathrm{h})$ & $4.5 \pm 0.8$ & $4.4 \pm 0.7$ \\
\hline \multicolumn{3}{|l|}{ Physical activity } \\
\hline Total activity score ${ }^{+}$ & $10147 \pm 5030$ & $9919 \pm 4291$ \\
\hline $\begin{array}{l}\text { Training distance last } 2 \text { weeks } \\
\text { before walking event }(\mathrm{km})\end{array}$ & $37 \pm 34^{*}$ & $68 \pm 49^{*}$ \\
\hline $\begin{array}{l}\text { Average heart rate at day } 1 \\
\text { (bpm) }\end{array}$ & $114 \pm 14$ & $113 \pm 11$ \\
\hline Exercise intensity at day $1(\%)$ & $67 \pm 8$ & $66 \pm 7$ \\
\hline
\end{tabular}

Table 2: Baseline characteristics of patients with IBD and control group with no IBD. Continuous data are shown as mean $\pm \mathrm{SD}$, significance is tested with the t-test. Categorial data are shown as an absolute amount (percentage), the significance is tested with the Pearson chi-square test. ${ }^{*} p<0.05 ;{ }^{\S}$ Education level: no education, primary or lower vocational education and lower general secondary education (low);secondary vocational education and higher general secondary education (middle); higher vocational education and university (high). ${ }^{+}$Total activity score represents intensity factors per activity multiplied by the minutes per week spent on each activity as derived from the SQUASH. Abbreviations: IBD = inflammatory bowel disease; bpm = beats per minute. SQUASH = Short Questionnaire to Assess Health enhancing physical activity

\section{Hosted file}

image1.emf available at https://authorea.com/users/354360/articles/477962-refractoryneutrophils-and-monocytes-in-patients-with-inflammatory-bowel-disease-after-repeated- 
bouts-of-prolonged-exercise

Figure 1: Responsiveness of innate immune cells to the bacterial/mitochondrial stimulus fMLF in IBD $(\mathbf{N}=18)$ versus non-IBD patients $(\mathbf{n}=\mathbf{1 9})$. Responsiveness is determined by flowcytometric analysis of the cellular activation markers $C D 10(\boldsymbol{A}), C D 11 b(\boldsymbol{B}-\boldsymbol{D}), C D 35(\boldsymbol{E}-\boldsymbol{G})$ and the adhesion marker CD62L $(\boldsymbol{H}-\boldsymbol{J}) . M F I$ is compared between study groups at day 0 and after 3 consecutive days of prolonged moderate exercise with the Mann-Whitney $U$ test. Data are presented as scatter plot with median and interquartile range. Abbreviations: $I B D=$ inflammatory bowel disease; $M F I=$ Median fluorescent intensity; $A U=$ arbitrary units.

\section{Supplemental figure 1}
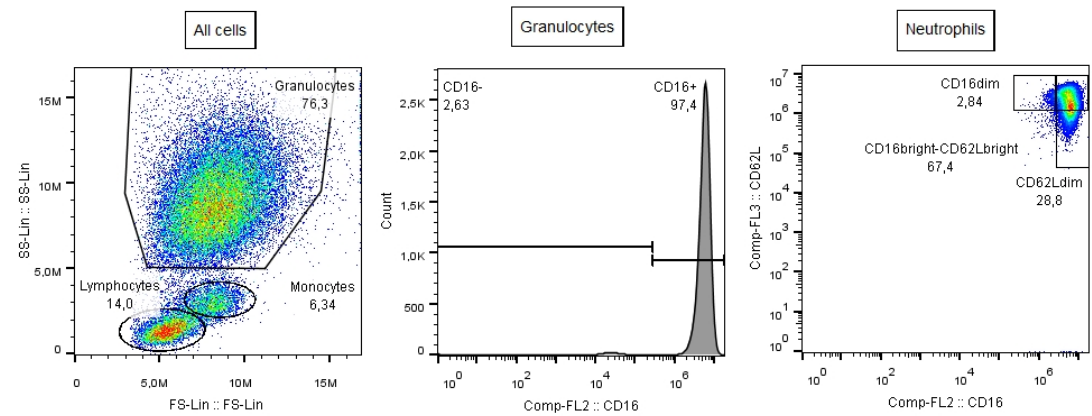

Gating strategy as performed in FlowJoß analysis software. The forward- and side scatter plot was first made to set gates for the lymphocyte- monocyte- and PMN populations (left). In the right plot, the CD16 expression of the PMN population is displayed. Gate was set to distinguish neutrophils CD16+ and eosinophils CD16-. 